\title{
Small-animal PET registration method with intrinsic validation designed for large datasets
}

\author{
J Pascau, Member, IEEE, J D Gispert, M Soto-Montenegro, A Rodríguez-Ruano, V García-Vázquez, A Udías, JJ \\ Vaquero, Senior Member, IEEE, M Desco
}

\begin{abstract}
We present a procedure to validate the results of small animal Positron Emission Tomography (PET) image registration by means of consistency measures. Small animal 2Deoxy-2-[F-18]fluoro-D-glucose (FDG) PET studies do not show the same intensity distribution even for images acquired in similar conditions, as the resulting image is influenced by several variables which are not always completely under control. Because of these difficulties, the results from automatic registration methods have to be visually inspected to detect failures. We propose a method to automate this validation process. Two reference images from the dataset are selected by an expert user avoiding images with poor contrast, animal movement or low quality, and both are co-registered using anatomical landmarks. All the remaining images in the dataset are then registered to every reference with an automatic two-step algorithm based on Mutual Information. The known transformation relating both references allows measuring the registration consistency, which is a good estimator of the accuracy of the alignment process, for every image in the dataset. This value can be used to assess the quality of the registration and therefore detect the incorrect results. We have applied this validation process on a large dataset of 120 FDG-PET rat brain images obtained with a rotating PET scanner. The registration consistency was calculated for every image in the dataset and values below $1.65 \mathrm{~mm}$ (PET image resolution) were considered as successful registrations. 116 images were correctly registered with an average error of $0.839 \mathrm{~mm}$, while in four images the proposed method detected a registration failure. Two of these failures were due to very low image quality and these studies were discarded from the study, while the other two were correctly aligned after applying a manual pre-alignment step. Our approach requires minimal user interaction and provides automatic assessment of the registration error, making it unnecessary to visually inspect and check every registration result.
\end{abstract}

\section{INTRODUCTION}

Small animal PET studies can be analyzed using statistical parametric methods, in order to assess group differences

Manuscript received November 22, 2007. This work was supported by projects CIBER CB06/01/0079 (Ministerio de Sanidad y Consumo) and CDTEAM (CENIT program, Ministerio de Industria).

J Pascau, M Soto-Montenegro, A Rodríguez-Ruano, V García-Vázquez, A Udías, JJ Vaquero, and M Desco are with Unidad de Medicina y Cirugía Experimental, Hospital General Universitario Gregorio Marañón, Madrid, Spain (telephone +34 915866678, e-mail jpascau@mce.hggm.es).

J D Gispert is with the Institut d'Alta Tecnologia, Parc de Recerca Biomèdica de Barcelona, Barcelona, Spain. without previously defining regions of interest for quantification. These methods require that all images are aligned thus allowing voxel-to-voxel comparisons. Registration of these type of images poses some difficulties due to the differences in intensity distribution even for images acquired in similar conditions, because the resulting scan is influenced by several variables which are not always completely under control, like blood glucose level previous to the injection or animal stress during uptake. Automatic registration methods based on information theory have been tested on small animal data with acceptable results $[1,2]$, but even with these methods the main drawback of the alignment process as a whole is that no measurement of the registration error can be obtained. Provided that not all the studies are always successfully registered with the automatic method, every aligned image pair must be visually checked and validated.

In this paper we propose a procedure to validate the registration results by means of objective consistency measurements [3]. The user selects two reference images and manually co-registers them. The whole dataset is then automatically registered to both references, and a composed transformation calculated for every image allows estimating the error of the registration process. This approach has been tested on a large dataset of small animal FDG-PET scans to show that it can detect registration failures avoiding the need to visually check the registration quality for every image.

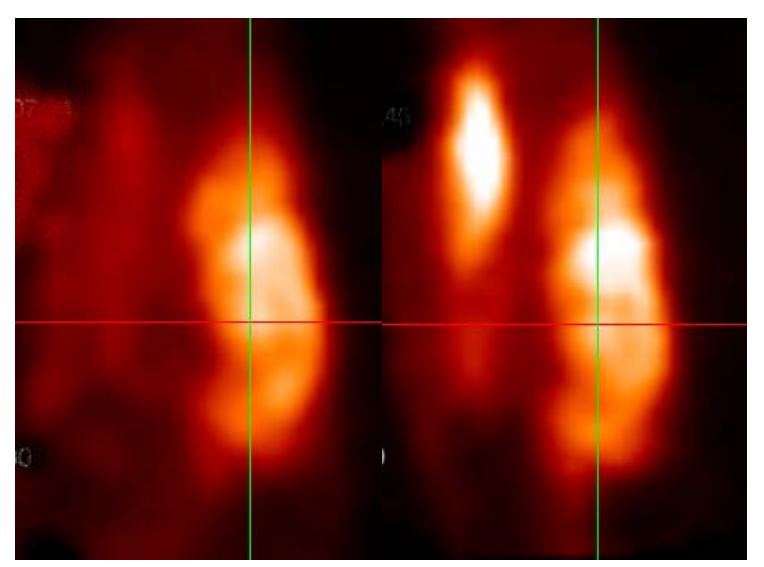


Fig. 1. Sagittal sections of two FDG-PET rat brain images (nose up). Differences in contrast are observed: both images show glucose metabolic activity in the brain, but structures outside the brain do not display similar activity in both images. Slight animal condition differences during FDG update are responsible for these differences.

\section{MATERIALS AND METHODS}

120 FDG-PET scans of male Dark Agouti rats were acquired using a rotating PET scanner [4]. Images were acquired for $60 \mathrm{~min}$ after a $30 \mathrm{~min}$ uptake, with animals under anaesthesia (isoflurane). Acquired data were reconstructed with 3DRP algorithm, obtaining images with [55,55,55] matrix size and voxel size $0.8145 \mathrm{~mm}$ isotropic. These data were part of a study on the cerebral effects of MDMA on cerebral metabolism. Figure 1 shows two images belonging to the dataset.

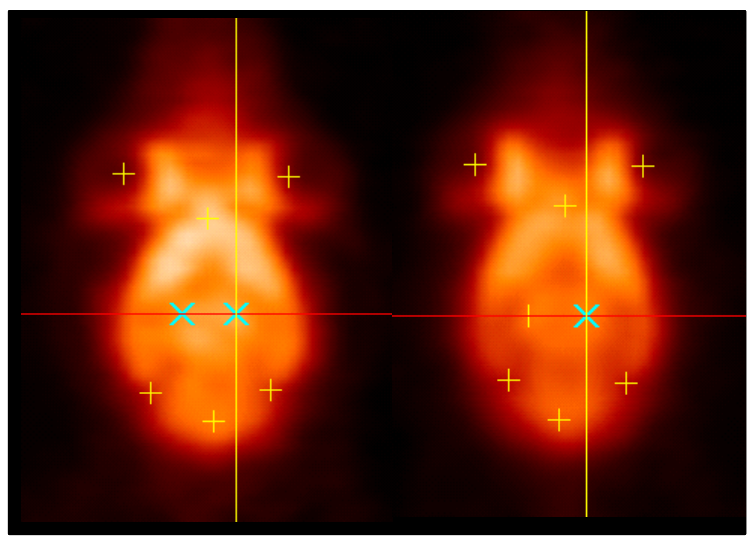

Fig. 2. Reference images selected by the user, with several anatomical landmarks used for manual registration.

Two reference images $\left(r_{1}\right.$ and $\left.r_{2}\right)$ were selected by an expert user avoiding images with poor contrast, animal movement or low quality. These reference images were co-registered using 5 pairs of anatomical landmarks (fig. 2), afterwards calculating the rigid transformation following the method proposed in [5]. All the images in the dataset were then registered to every reference with an automatic algorithm that optimized Normalized Mutual Information [6] with the following implementation details: multiresolution strategy with two sub-sampling steps (the first one subsamples every $[4,4,2]$ voxels in $[\mathrm{x}, \mathrm{y}, \mathrm{z}]$ directions, and the second every $[2,2,1])$; joint histogram estimation using Partial Volume Interpolation [7] with 64 bins; Powell optimization [8]. The algorithm worked on a two-step manner: in the first step (low resolution) the cost function is optimized using the whole reference image, while in the high resolution step the brain of the reference image is masked (only voxels inside the mask are used to calculate the cost function) in order to obtain the best quality in the structure of interest (the brain tissue). To avoid interpolation artifacts that are quite common when deriving information theory measures from the joint histogram [9], reference image was resampled, changing its matrix size from $[55,55,55]$ to $[65,65,65]$. The whole automatic registration process is depicted in fig. 3 .

To automate the validation process, we measure the registration consistency, following the idea initially proposed by Woods [3]. Briefly, it assesses the registration quality by co-registering three images, composing the transformations obtained and measuring the difference between this composed transformation and the identity.

Every image $I_{j}$ in the dataset was registered to every reference image $\left(r_{1}\right.$ and $\left.r_{2}\right)$ obtaining transformations $T_{I j r l}$ and $T_{I j r 2}$ and the associated homogeneus matrices $\left(M_{I j r l}\right.$ and $\left.M_{I j r l}\right)$. In the absence of registration error, being $\circ$ the composition of transformations, $T_{I j r 1} \circ T_{r I r 2} \circ T_{r 2 I j}$ should correspond to the identity transformation. Thus, the difference between $M_{I j r 1} \cdot M_{r l r 2} \cdot M_{r 2 I j}$ and the identity matrix $I$ estimates the registration error:

$$
\Delta M_{j}=M_{I j r 1} \cdot M_{r 1 r 2} \cdot M_{r 2 I j}-I
$$

In an attempt to represent this error with a single number, we selected a bounding box containing our volume of interest (the brain), and calculated the average error in the eight vertices of this box $\left(p(i)=\left[x_{i}, y_{i}, z_{i}\right] i=1 . .8\right)$ :

$$
R C I_{j}=1 / 8 \sum_{i=1 . .8}\left\|\Delta M_{j} \cdot p(i)\right\|
$$

This Registration Consistency Index $\left(R C I_{j}\right)$ measures the registration consistency for image $I j$ and reference images $r_{l}$ and $r_{2}$. To classify the registration of image $I_{j}$ with both references as successful or not, the image resolution (1.65 $\mathrm{mm}$ ) is used as a threshold, in such a way that when the $R C I_{j}$ is lower than this value the registration is classified as Successful and for misalignments larger than image resolution the registration result is labelled as Failure.

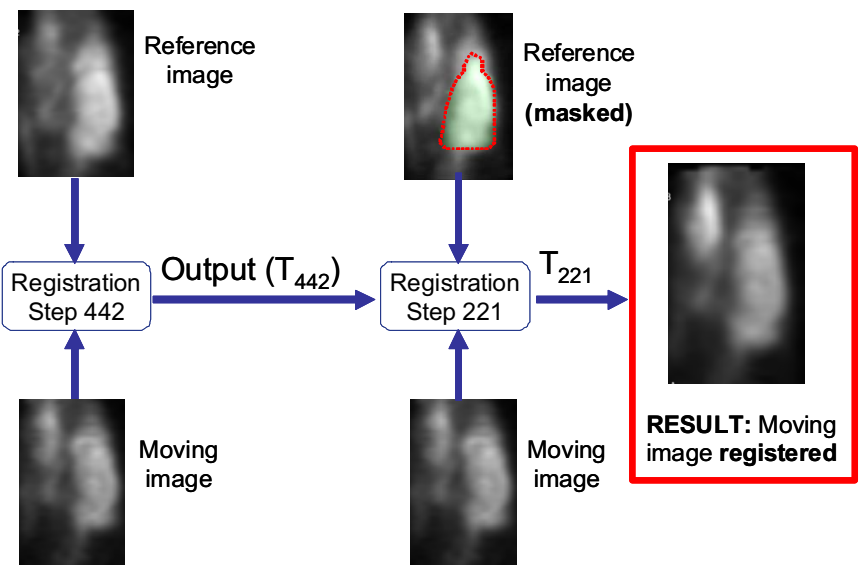


Fig. 3. Scheme for the automatic registration process. The whole reference image is used in the first multiresolution step, and the output transformation $\left(\mathrm{T}_{442}\right)$ is then the input for the last step, in which the reference image is masked in order to obtain best registration quality inside the brain.

\section{RESULTS AND DISCUSSION}

The proposed validation method was applied to the 120 images in the dataset. In four cases the consistency index $R C I$ showed a value above the defined threshold, and these registration results were classified as failures. The average error for the 116 images correctly registered was $0.839 \mathrm{~mm}$ $(\max 1.1304 \mathrm{~mm}, \min 0.5281 \mathrm{~mm})$, indicating a high registration quality in terms of the PET resolution $(1.65 \mathrm{~mm})$, Every case was registered in 4 secs. on average, and the whole dataset needed 16 mins. to be registered with both references. The registration and validation process has only two userdependent steps: reference image selection and brain segmentation in these two images. Fig. 4 shows four representative images correctly registered with one reference.

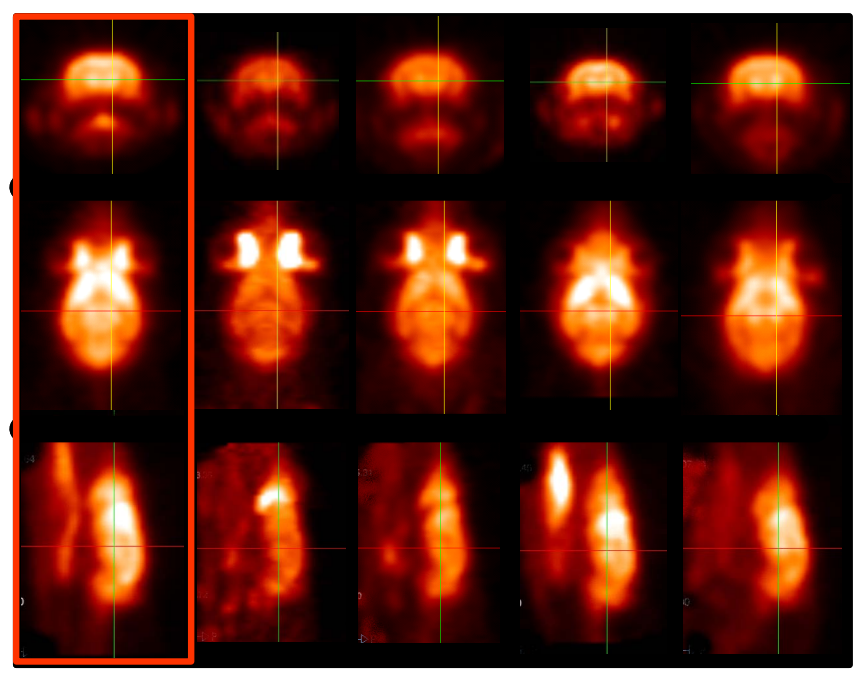

Fig. 4. Axial, coronal and saggittal views of several images correctly registered to the referente image (first column). Axes position is the same for all the studies.

The four images classified as incorrect registrations according to the consistency index were visually examined. Two of them were scans with acquisition errors that produced reconstructed images extremely noisy, in which no structure could be identified. These scans were discarded from the study. The other two incorrectly registered images showed a very high intensity area at the catheter insertion point, and consequently the brain structure showed low intensity values in comparison. This high dynamic range affects the histogram shape and makes automatic registration more difficult. In both cases, a manual pre-alignment step followed by automatic registration produced finally a valid result.

Our approach requires minimal user interaction and provides automatic assessment of the registration error, making it unnecessary to visually inspect and check every registration result. This method will thus facilitate the accurate, objective and rapid analysis of large groups of rodent PET images.

\section{ACKNOWLEDGMENT}

The authors thank SUINSA Medical Imaging Engineering department for their support during the development of the rotating PET scanner

\section{REFERENCES}

[1] Rubins, D.J., et al., Development and evaluation of an automated atlasbased image analysis method for microPET studies of the rat brain. Neuroimage, 2003. 20(4): p. 2100-18.

[2] Vaquero, J.J., et al., PET, CT, and MR image registration of the rat brain and skull, in Nuclear Science, IEEE Transactions on. 2001, Practical. p. 1440-1445.

[3] Woods, R.P., et al., Automated image registration: I. General methods and intrasubject, intramodality validation. J Comput Assist Tomogr, 1998. 22(1): p. 139-52.

[4] Vaquero, J., et al., Preliminary results of the small animal rotational positron emission tomography scanner. Molecular Imaging and Biology, 2004. 6(2): p. 102.

[5] Arun, K.S., T.S. Huang, and S.D. Blostein, Least-Squares Fitting of Two 3-D Point Sets. IEEE Trans. Pattern Anal. Machine Itell., 1987. PAMI-9(5): p. 698-700.

[6] Studholme, C., D.L.G. Hill, and D.J. Hawkes, An overlap invariant entropy measure of $3 D$ medical image alignment. Pattern Recognition, 1999. 32(1): p. 71-86.

[7] Maes, F., et al., Multimodality image registration by maximization of mutual information. IEEE Trans Med Imaging, 1997. 16(2): p. 187-98.

[8] Press, W.H., et al., Numerical Recipes in C. Second ed. 1992: Cambridge University Press.

[9] Pluim, J.P., J.B. Maintz, and M.A. Viergever, Interpolation artefacts in mutual information based image registration. Comput Vis Image Underst, 2000. 77(2): p. 211-232. 